\title{
The effect of family history of diabetes on sleep quality, depression, and anxiety in adult males with diabetes
}

\author{
Carolyn A. Fan ${ }^{1}$, Lindsey Sattler ${ }^{1 *}$, Christopher Parkinson ${ }^{1}$, Boby G. Thekadeth ${ }^{2}$, \\ Sant P. Singh ${ }^{2}$, Lawrence C. Perlmuter ${ }^{1}$ \\ ${ }^{1}$ Department of Psychology, Rosalind Franklin University of Medicine and Science, North Chicago, USA; \\ *Corresponding Author: lindsey.sattler@my.rfums.org \\ ${ }^{2}$ Captain James A. Lovell Federal Health Care Center, North Chicago, USA
}

Received 28 October 2012; revised 26 November 2012; accepted 4 December 2012

\section{ABSTRACT}

Study Objectives: This study evaluated how a family history of diabetes moderates the effects of diabetes on anxiety, depression, as well as sleep quality. Design: Outpatients with diabetes responded to questionnaires on family history of diabetes, sleep quality, anxiety and depression. Participants: Participants were recruited from the Captain James A. Lovell Federal Health Care Center in North Chicago, Illinois. All participants were adult males $(n=33)$ with ages ranging from 50 to 88 and were diagnosed with type 1 or type 2 diabetes. There were 8 subjects without a family history of diabetes and 25 subjects with family history of diabetes. Results: In comparison to those with a family history of diabetes, patients without a family history of diabetes were found to experience a poorer quality of sleep, evaluated on the Pittsburgh Sleep Quality Index [PSQI] $(t=3.62, p=0.001)$. Anxiety, depression, family history and the Interaction of family history with depression were found to be significant predictors of sleep quality $(p<0.05)$. Conclusions: Patients without a family history of diabetes exhibit more anxiety and depression and have more sleep problems than those patients who have a family history of diabetes. Presumably a family history of diabetes better informs and prepares these patients for the many unique problems associated with diabetes thereby decreasing disease related anxiety and depression.

Keywords: Diabetes; PSQI; Sleep Quality; Anxiety; Depression; Family History

\section{INTRODUCTION}

According to the American Diabetes Association [ADA] [1], diabetes mellitus is a chronic disease in which patients among other debilitations suffer from hyperglycemia due to irregular production of insulin or the development of insulin resistance. In recent years, type 2 diabetes has become more common in the developed world. In the United States, the number of adult diabetic patients increased $61 \%$ between the years 1991 and 2000. This is likely due to changes in lifestyle [2], such as poor diet and lack of exercise. A family history of the disease also puts many of these patients at additional risk for developing type 2 diabetes [3].

A patient's family history can affect how their offspring manage and cope with their health status and in this instance diabetes [4]. That is, subjects with a negative family history of diabetes exhibited poorer glycemic control as disease duration increased, while patients with a positive family history showed relatively stable glycemic control over time [5]. Perhaps patients with a positive family history were more vigilant in monitoring their glycemic control.

In families with multigenerational occurrences of diabetes, the initial exposure to diabetes may be through the family. That is, family members with diabetes can in this instance have a positive impact on behaviors of the successive generations who are diagnosed with diabetes [6].

Rubin, Peyrot, \& Saudek [7] showed that a week-long educational program for patients with diabetes was found to boost self-esteem and self-efficacy while reducing anxiety and depression over the course of a six month period. Decreases in anxiety and depression are particularly important for patients with diabetes because compared to the general population, depression and anxiety are elevated [2]. Thus, in patients with diabetes, knowledge and even vicarious experience through family his- 
tory could provide a key learning factor in successful management of depression and other symptoms associated with diabetes.

Sleep problems are also prevalent among patients with diabetes. Plantinga, Rao, \& Schillinger [8] showed that diabetes was positively correlated with sleep disturbances such as restless leg syndrome, sleep apnea, and nocturia in $90 \%$ of subjects. Even in healthy adults, sleep deprivation has also been linked to impaired glucose tolerance [9]. When sleep was restricted to 4 hours for 6 nights, subjects experienced a $40 \%$ decrease in glucose tolerance. These effects were reversed, however, with sleep recovery [9]. An additional study also demonstrated an association between sleep complaints such as sleep apnea, insomnia and daytime sleepiness and insulin resistance in patients without a diagnosis of diabetes [10]. This observation implicates a link between sleep and insulin resistance $[9,10]$.

Sleep quality, which is assessed with the Pittsburgh Sleep Quality Index [PSQI], has also been compromised in many patients with diabetes [11]. It was found that $69 \%$ of patients with diabetes had a global PSQI score greater than five (mean 7.08, SD 3.89), which indicated that they had poor sleep quality [11]. Seligowski et al. [12] showed that poor sleep quality, which was evaluated on the PSQI scale, was positively associated with depression and anxiety in patients with diabetes. Results also indicated that sleep quality had an indirect effect on quality of life, which measures satisfaction with treatment, perceived effectiveness of treatment, worry about social/vocational issues, and worry about the future effects of diabetes [12]. Research has also investigated the effects of aging on sleep quality. It does not seem that age alone affects sleep quality and sleep problems are not an inevitable part of aging. Alternatively, sleep problems in older adults are often comorbid with underlying medical or psychiatric illnesses [13].

In the present study, we examined the association between family history of diabetes and depression, anxiety, and sleep quality, all of which are potential problems in patients with diabetes. Also, patients with a family history of diabetes have been shown to better manage their diabetes symptoms [5]. This may be the result of increased knowledge gained from their experience with parents [6]. A week-long educational program on diabetes care also reduced the levels of depression and anxiety in patients [7]. Therefore, the insight and familiarity that comes from family history could also serve to reduce psychosocial symptoms. Since depression and anxiety were linked to poor sleep quality [12], a positive family history of diabetes may be associated with a higher quality of sleep. Therefore, it is hypothesized that patients with a family history of diabetes should have a better quality of sleep and fewer symptoms of anxiety and depression.

\section{METHODS}

\subsection{Participants}

Subjects were recruited at the Captain James A. Lovell Federal Health Care Center in North Chicago, Illinois. The resulting sample was comprised of 33 adult male subjects with type 1 and type 2 diabetes, with ages ranging from 50 to 88 . This wide age range is representative of the sample and is comparable in those with a family history of diabetes (mean $=66.8$ ) and without a family history of diabetes (mean $=65.3$ ). There were 29 Caucasian subjects, and the remaining subjects were African American, Hispanic, Asian, or other. Within the study, 25 people had a family history of diabetes, and 8 did not. The risk of type 2 diabetes increases if relatives also have the disease, which may contribute to the low number of subjects without a family history of diabetes. The overall mean HbAlc value was 7.56 (Std Dev 1.30). Subjects completed the Mini Mental State Examination [MMSE] and those who scored below a 24 were not allowed to continue. This criterion did not exclude any subjects [14].

\subsection{Procedure}

Patients were asked to participate in a questionnaire about metabolic control, demographic data, psychosocial function, and sleep quality. Patients were asked about their family history of diabetes. If one or more parents had diabetes, they were defined as having a positive family history. Subjects were then asked to evaluate their anxiety level using the 4 Item Perceived Stress Scale [PSS-4] [15]. Depression was evaluated on the Zung Self-Rating Depression Scale. Scores on the Zung scale range from 20 to 80 [16]. The questionnaire was presented to patients in a low stress environment when they were receiving a routine checkup.

Lastly, the PSQI was administered as part of the questionnaire. Responses on the PSQI were used to determine the overall sleep quality of the individuals over the past month. Patients rated their sleep duration, sleep disturbances, sleep latency, trouble staying awake, problems with enthusiasm for staying awake, sleep efficiency, sleep quality, and sleep medications. Each factor was evaluated by one of nine questions. The answer options used were slightly modified; it stated, "Never or a little of the time, some of the time, good part of the time, most or all of the time". Each answer resulted in a score from zero to three. A cumulative score was then calculated, which ranged from 0 (best sleep quality) to 21 (worst sleep quality). Scores above five indicate poor sleep quality [2].

\subsection{Stastical Analysis}

SPSS Software 12.0 was used to analyze the data. A 
significance level of $p<0.05$ was used for all analyses. Multiple regression and t-tests were utilized.

\section{RESULTS}

A scatter plot displayed depression and PSQI scores for those with and without a family history. For those without a family history, sleep quality and depression seem more strongly related (Figure 1). A single subject without a family history had a PSQI score of 19 (Figure 1), while the average score on the PSQI for subjects without family history was 10.9 (Std Dev 4.8) and when this subject was removed from analysis, results remained significant.

An independent t-test indicated that those with a positive family history exhibited better sleep quality $(t=3.62$, $p=0.001$ ). A linear multiple regression was used to determine how sleep quality was associated with the following variables: anxiety, depression, family history of diabetes, and the Interaction between Depression X Family History (Table 1). The Interaction showed that the effects of depression and sleep quality are moderated by family history $(p<0.05)$. Although anxiety and depression were correlated with each other $(p=0.002)$, when the Interaction between Family History X Anxiety was examined it was not found to be a significant predictor of sleep quality even though the Interaction between Family History X Depression was significant. The order of entry for depression and anxiety in the linear regression model did not importantly alter the results.

The relationship between age, depression, anxiety and sleep quality was also evaluated. Bivariate correlations indicated that age was not correlated with depression or

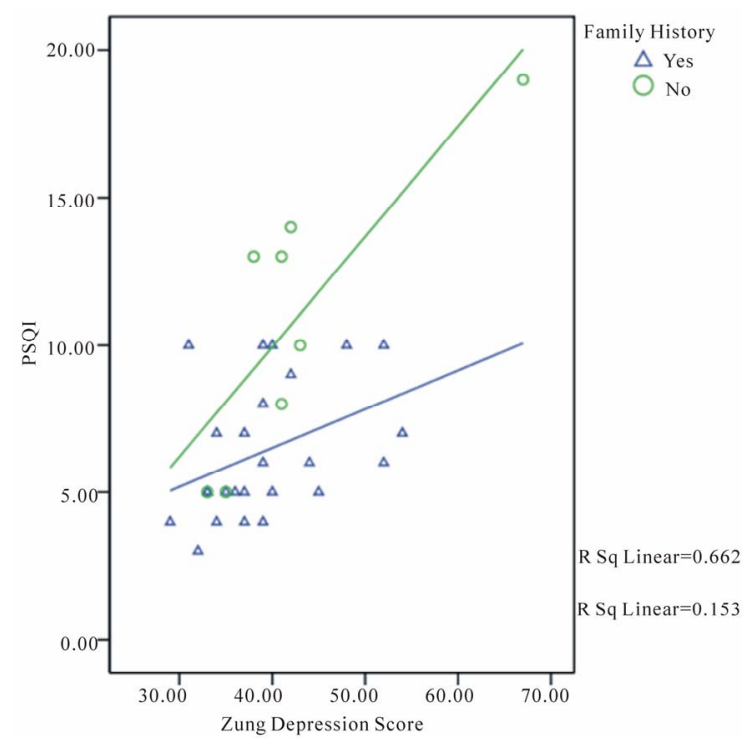

Figure 1. Cumulative PSQI score (Lower score indicates better sleep quality) and depression in individuals with positive or negative family history of diabetes.
Table 1. Hierarchical multiple regression model: predictors $=$ Anxiety, Depression, Family History (yes = 1, no = 2), Interaction between Depression X Family History; dependent variable = PSQI (Lower score indicates better sleep quality).

\begin{tabular}{cccc}
\hline Predictors & $\mathrm{R}^{2}$ Change & Beta & F Change \\
\hline Anxiety & 0.44 & 0.66 & $24.30^{*}$ \\
Depression & 0.08 & 0.33 & $5.04^{\dagger}$ \\
Family History & 0.14 & 0.39 & $11.88^{*}$ \\
Depression X Family History & 0.06 & 1.45 & $5.48^{\dagger}$ \\
\hline
\end{tabular}

${ }^{*} P<0.01,{ }^{\dagger} P<0.05$.

Table 2. Hierarchical multiple regression model: predictors = Anxiety, Depression, Interaction between Age X Anxiety, Interaction between Age $\mathrm{X}$ Depression; dependent variable = PSQI (Lower score indicates better sleep quality).

\begin{tabular}{cccc}
\hline Predictors & $\mathrm{R}^{2}$ Change & Beta & F Change \\
\hline Anxiety & 0.44 & 0.66 & $24.30^{*}$ \\
Depression & 0.08 & 0.33 & $5.04^{\dagger}$ \\
Age X Anxiety & 0.04 & -0.80 & 2.33 \\
Age X Depression & 0.01 & -0.29 & 0.71 \\
\hline
\end{tabular}

${ }^{*} P<0.01,{ }^{\dagger} P<0.05$.

anxiety in either group of subjects. There was, however, a negative correlation between age and sleep quality ( $p=$ 0.001 ) seen in subjects with a family history of diabetes. This indicates that as age increases, sleep quality is actually slightly improved. This effect was not seen in those without a family history of diabetes. A linear multiple regression examined the relationship between sleep quality and anxiety, depression, the Interaction between anxiety and age, and the Interaction between depression and age (Table 2). The Interaction between age and depression and age and anxiety were not found to be significant predictors of sleep quality.

\section{DISCUSSION}

This study indicated that family history plays an important role in psychological and sleep behaviors in patients with diabetes. Sleep quality was poorer in those with a negative family history of diabetes and the strength of the relationship with depression was stronger in the absence of a positive family history. Several factors were predictors of sleep quality, including anxiety, depression, family history, and the Interaction of Family History X Depression.

Possibly, the knowledge and familiarity gained from a family history of diabetes helps patients to better cope with the disease. Positive family setting provides a unique opportunity to have a first-hand experience in managing chronic diseases [6]. Previous research has shown that patients with a family history of diabetes are more successful in managing metabolic symptoms in com- 
parison to those without a family history [5]. In the current study, patients who have familial experiences with diabetes exhibit better sleep quality. Perhaps, the knowledge gained from having a family member with diabetes generates a perception of control over their disease [6] thereby promoting a stronger sense of ease and enabling better sleep quality.

Research has indicated that an increase in knowledge of diabetes care can lessen symptoms of anxiety and depression [7]. Since family history may be tied to greater knowledge [6], it seems as though a positive family history could also indirectly reduce anxiety and depression. Seligowski et al. [12] showed that anxiety and depression are correlated with a poorer quality of sleep in patients with diabetes. The results from this present study supported these findings. It was found that a positive family history, along with less depression and anxiety, were significant predictors for better sleep quality.

Additionally, while increased age does not seem to impact depression and anxiety, it was found to have a positive effect on sleep quality in those with a family history of diabetes. While these findings are surprising it seems as though age does not independently impact sleep quality. Sleep problems seen in older individuals can often be explained by other underlying medical or psychological problems, not by age itself [13]. In the present study the increased sleep quality in those with a family history of diabetes may be related to increased knowledge that also seems to reduce anxiety and depression.

The main limitation of this study is sample size. There were 33 subjects, with only 8 having no family history of diabetes. While this is a small sample size, there is a significant difference in results of those with a family history of diabetes and those without. There is also wide age range in the subjects. This range is present in both groups of subjects and is representative of the subject group, but a smaller age range would help provide more focused results. Despite these limitations, this study provides a foundation for exploring the effect of family on sleep quality, depression, and anxiety.

\section{REFERENCES}

[1] American Diabetes Association (1992) Office guide to diagnosis and classification of diabetes mellitus and other categories of glucose intolerance. Diabetes Care, 15, 3-4.

[2] Anderson, R.J., Freedland, K.E., Clouse, R.E. and Lustman, P.J. (2001) The prevalence of comorbid depression in adults with diabetes. Diabetes Care, 24, 1069-1078. doi:10.2337/diacare.24.6.1069

[3] Wing, R.R., Venditti, E., Jakicic, J.M., Polley, B.A. and Lang, W. (1998) Lifestyle intervention in overweight individuals with a family history of diabetes. Diabetes Care, 21, 350-359. doi:10.2337/diacare.21.3.350
[4] Elfant, E., Gall, E. and Perlmuter, L.C. (1999) Learned illness behavior and adjustment to arthritis. Arthritis Care and Research, 12, 411-416. doi:10.1002/1529-0131(199912)12:6<411::AID-ART9>3 .0.CO;2-B

[5] Perlmuter, L.C., Singh, S.P., Gabhart, J.M., Pungan, R. and Siedlarz, M. (2008) Parents matter: Intergenerational Influences of diabetes mellitus on glycemic control and morbidity in older type 2 diabetic males. Experimental Aging Research, 34, 138-151. doi:10.1080/03610730701876961

[6] Scollan-Koliopoulos, M., O'Connel, K. and Walker, E. (2005) The first diabetes educator is the family: using illness representation to recognize a multigenerational legacy of diabetes. Clinical Nurse Specialist, 19, 302-307. doi:10.1097/00002800-200511000-00009

[7] Rubin, R.R., Peyrot, M. and Saudek, C.D. (1989) Effect of diabetes education on self-care, metabolic control, and emotional well-being. Diabetes Care, 2, 673-679. doi:10.2337/diacare.12.10.673

[8] Plantinga, L., Rao, M.N. and Schillinger, D. (2012) Prevalence of self-reported sleep problems among people with diabetes in the United States, 2005-2008. Preventing Chronic Disease, 9, 110-244.

[9] Spiegel, K., Leproult, R. and Van Cauter, E. (1999) Impact of sleep debt on metabolic and endocrine function. Lancet, 354, 1435-1439. doi:10.1016/S0140-6736(99)01376-8

[10] Pyykkonen, A., Isomaa, B., Pesonen, A., et al. (2012) Subjective sleep complaints are associated with insulin resistance in individuals without diabetes. Diabetes Care, 35, 2271-2278. doi: $10.2337 / \mathrm{dc} 12-0348$

[11] Buysse, D.J., Reynolds, C.F., Monk, T.H., Berman, S.R. and Kupfer, D.J. (1989) The Pittsburgh Sleep Quality Index (PSQI): A new instrument for psychiatric research and practice. Psychiatry Research, 28, 193-213. doi:10.1016/0165-1781(89)90047-4

[12] Seligowski, A.V., Kaiser, K.A.P., Niles, B.L., Mori, D.L., King, L.A. and King, D.W. (2012) Sleep quality as a potential mediator between psychological distress and diabetes quality of life in veterans with type 2 diabetes. Journal of Clinical Psychology, 1-11. doi:10.1002/jclp.21866

[13] Ancoli-Israel, S., Ayalon, L. and Salzman, C. (2008) Sleep in the elderly: Normal variations and common sleep disorders. Harvard Review of Psychiatry, 16, 279-286. doi:10.1080/10673220802432210

[14] Folstein, M.F., Folstein, S.E. and McHugh, P.R. (1975) Mini-mental state. A practical method for grading the cognitive state of patients for the clinician. Journal of Psychiatric Research, 12, 189-198.

[15] Cohen, S., Kamarck, T. and Mermelstein, R. (1983) A global measure of perceived stress. Journal of Health and Social Behavior, 24, 385-396. doi:10.2307/2136404

[16] Zung, W.K. (1965) A self-rating depression scale. Arch Gen Psychiatry, 12, 63-70. doi:10.1001/archpsyc. 1965.01720310065008 